\title{
Revisiting the Belmont Report's ethical principles in internet-mediated research: perspectives from disciplinary associations in the social sciences
}

\author{
Icy Fresno Anabo ${ }^{1}$ (1) - Iciar Elexpuru-Albizuri ${ }^{1}$ (1) - Lourdes Villardón-Gallego ${ }^{1}([)$
}

Published online: 28 December 2018

(c) The Author(s) 2018

\begin{abstract}
The purpose of this article is to illuminate the conceptualisations and applications of the Belmont Report's key ethical principles of respect for persons, beneficence, and justice based on a document analysis of five of the most relevant disciplinary guidelines on internet research in the social sciences. These seminal documents are meant to provide discipline-specific guidance for research design and implementation and are regarded as key references when conducting research online. Our analysis revealed that the principles of respect and beneficence were explicitly conveyed in the documents analysed, offering nuanced interpretations on issues of informed consent, privacy, and benefits and risks as well as providing recommendations for modifying traditional practices to fit the online setting. However, the invocations of the principle of justice were rather implicit and reflect an important shift from the Belmont Report's protectionist ethical position towards more situational and dialogic approaches. With the rapidly evolving nature of internet technologies, this analysis is projected to contribute to the ongoing developments in research ethics in the social sciences by outlining the tensions and implications of the use of the internet as a methodological tool. We also seek to provide recommendations on how disciplinary associations can proceed to facilitate ethically sensitive internet research.
\end{abstract}

Keywords Internet research ethics · Online research ethics - Digital research ethics · Ethics guidelines for internet research · Belmont Report

\section{Introduction}

The internet has been converted into what Castells (2001) describes as the fabric of our lives, revolutionizing the way people's perceptions and interactions with their environment are reflected. In the field of social science research, it is viewed by many as a rapidly developing and massively potent methodological tool due to its role in shaping the dynamics, locations, and embodiments of interactions and meaning construction (Jones 2011; Orton-Johnson 2010; Parker et al. 2011; Savage and Burrows 2007; Townsend and Wallace 2016; Wilson et al. 2012).

However, the internet's use in social science research is fraught with ethical and legal challenges, some of which appear to be more complex than what traditional research

Icy Fresno Anabo

icyanabo@deusto.es

1 University of Deusto, Bilbao, Spain ethics can address. The multi-paradigmatic and fluid nature of the social sciences prompts it to remain adaptable to its changing object of inquiry, our society, which now involves the internet (Hine 2005). As Buchanan (2011) contends, the changes in spaces and technology afforded by the internet "has the potential to fundamentally disturb the extant models of human subjects research" (p. 83), paving the way for the creation of what she coins research ethics 2.0. Moreover, the implementation of research in online as opposed to face-to-face contexts raises several issues that warrant new models and approaches. Jones (2011) points out how the development of Web 2.0 platforms, including social media, has expanded possibilities for participation as well as ethical issues and dilemmas.

These developments and the concomitant ethical concerns they raise seem to be recognized by those involved in both academic and market research. As a result, scholars, university institutional review boards (IRBs), human research ethics committees (HRECs), research councils, and a variety of other public and private organizations have released 
guidelines pertaining to the ethical use of the internet. While there is a wealth of literature pertaining to internet research in general, there are still gaps to be filled. Firstly, the continuous advancement of technologies and the wider variety of data that can be harvested online require guidelines to be regularly revised to reflect the latest issues and debates that arise from this ever-changing field. Secondly, the applications of internet research ethics are disciplinespecific (Buchanan and Zimmer 2018) and will thus need to consider the different applications between fields. This article focuses on internet research ethics in the social sciences and aims to illuminate the applications and implications of ethical guidelines for the benefit of individuals who identify their research practice within this discipline.

Disciplinary associations, or learned societies, are one of the key entities in guiding researchers in the conduct of ethically sensitive studies. According to Beauchamp and Childress (1994), professional codes of conduct can play a significant role in reinforcing member identification and in upholding professional values. Within the realm of a specific discipline, they are particularly helpful when deciding on the study design and reflecting on the discipline-specific dilemmas that arise in the choice of methods. In the same vein, Platt (2015) describes how disciplinary associations have been an important source of new knowledge and ethical codes for the practice of professions, ultimately contributing to legitimizing the profession's knowledge claims, activities, and standards as well as the cooperation between its members, among others.

Identifying disciplinary associations' stance on ethical issues arising from the use of the internet as a methodological tool is thus deemed worthwhile, especially in social science research where boundaries are often loose. This paper aims to contribute to the growing literature on internet research by identifying the conceptualizations and applications of traditional ethical principles in the current disciplinary guidelines on internet-mediated research. Ultimately, our goal is to advance the interpretation and reinterpretation of ethical concepts when implementing studies online involving human participants. We begin this venture by clarifying the scope of internet-mediated research in the following section and discussing how the rise of the internet has provided challenges in the dominant understanding of the ethical principles of research.

\section{Types of internet-mediated research}

Nowadays, the internet serves as a tool for researchers to carry out a wide range of methods alongside its role as a subject of inquiry itself (Jones 2011; Markham and Buchanan 2012; Orton-Johnson 2010). This article focuses on the role of the internet as a medium and not as a subject for research, hence the term internet-mediated research. It refers to activities such as online surveys, web page content analysis, videoconferencing, e-conversations through social media sites, email, chatrooms, and discussion boards and/or blogs (Convery and Cox 2012). To further distinguish its varied forms, Hewson et al. (2003) categorise the internet's myriad of methodological functions as either primary or secondary internet research. The former includes online recruitment and data collection from human subjects while the latter involves the use of non-human resources such as digital books and journals. Traditionally, conducting primary internet research undergoes more stringent ethical procedures. According to Eysenbach and Till (2001), primary internet research may be grouped into three categories, namely passive analysis, active analysis, or a mix of these approaches. The difference among these categories lies in the subjects' knowledge that they are being studied and the researcher's level of participation in communications.

Active analysis refers to a generally participative approach in internet research that reveals the identity of the researcher and allows his or her participation in data creation. Meanwhile, passive analysis alludes to Lee's (2000) definition of unobtrusive methods and Roberts's (2015) covert research wherein researchers act as observers without direct interaction with the data's creator or source and participants are unaware that they are being observed for research purposes. These include social network analysis, hyperlink analysis, and analysis of "found" data (Hine 2011). This method also includes big data or blog mining and web scraping implemented in social media-based market research (ESOMAR and GBRN 2015). Eysenbach and Till (2001) further describe the existence of a mix between passive and active analyses wherein the researcher's identity is made known while his or her role is confined to a participant recruiter and data collector rather than a data contributor.

\section{Ethical human subjects research and internet technologies}

Regardless of the study's location in aforementioned categories, an important distinction is made between the use of the internet with documents (archival research) and people (human subject research) (Walther 2002). Historically, the concern for ethical research involving human subjects was a response to a series of human rights violations following the Second World War and the United States' Tuskegee Experiments, leading to the development of the earliest code of ethics embodied in the Nuremberg Code of 1947. This further led to the development of other seminal texts such as the United Nations Declaration of Human Rights in 1948, the Declaration of Helsinki in 1964, and the National Commission for the Protection of Human Subjects of Biomedical and Behavioral Research's (hereon referred to as National Commission) Belmont Report in 1979 (Markham 
and Buchanan 2015; Buchanan 2011; Eynon et al. 2008). The Belmont Report has since served as a key reference in evaluating the ethical sensitivity of studies involving human participants. Specifically, the Belmont Report recommends that informed consent be sought, that benefits and risks be evaluated, and the selection, representation, and the burden of participation be fair and equitable. The negative effects of the lack of these principles' application would translate to coercion, harm, and undue involvement of vulnerable and burdened subjects.

Several authors argue that its biomedical roots are being extended and applied to unfit contexts (Beauchamp and Childress 1994; Gunsalus et al. 2006) including social and behavioral research (Siebert et al. 2002). This "mission creep" is seen to be problematic when applied to non-biomedical contexts because of the differences in the nature of inquiry and the methods employed (Gunsalus et al. 2006). Vilar Martín (2013) argues that the socio-educational fields should adapt the bioethical proposal of the Belmont Report in the context of social research. Nonetheless, the Belmont Report retains its authoritative status in ethical decisionmaking where its principles of respect for persons, beneficence, and justice are regarded by several authors as the cornerstone of ethical decision-making with human actors (Markham and Buchanan 2012; Armstrong 2003; Buchanan 2011). Ongoing discussions on research ethics use these principles to gauge ethical sensitivity, making them a good point of departure for exploring research practices when using the internet.

When the internet came into the picture and was utilised by researchers as a methodological tool, the existing model for ethics on human subjects research was further challenged. The Association of Internet Researchers (AoIR) (Markham and Buchanan 2012), together with Buchanan (2011) and Bassett and O'Riordan (2002), render the continuing use of the term human subjects inadequate when thinking about the internet and the social behaviors that it shapes and facilitates. While there are numerous configurations of what constitutes human subjects research, it is most commonly identified in regulatory contexts as involving the interaction with individuals and the use and archive of personally identifiable data (Frankel and Siang 1999; Moreno et al. 2013). However, Bassett and O'Riordan (2002) also suggest a more nuanced application of the human subject ethics model to internet research in light of its textual, language-focused, and sometimes detached nature from its creator that challenge the more widespread notion of the internet as merely a spatial venue with online information acting as mere extensions and representations of the individuals' selves. The British Sociological Association's (BSA) document further quotes Kozinets (2015) who argues that "the Internet is actually textlike and spacelike [and] these qualities exist both separately and simultaneously" (2015, p. 135).
While we do not wish to neglect the equally valid contention of the textual, and ergo distinct treatment, owed to information found online, our decision to apply the Belmont Report's principles of respect, beneficence, and justice stems from our acceptance of the looser definition of human subjects research, wherein the use and archive of personal information found online is regarded to be subject to ethical scrutiny. Through this article, we aim to illuminate how the Belmont Report's ethical principles were interpreted and applied in light of five disciplinary associations' guidelines on internet-mediated research as well as identify areas for their further development in future versions.

\section{Methods}

Ethics pertaining to human subjects is wide in scope and is subject to overarching human rights principles. Professional boards, research institutions, and even universities have developed and adapted a set of codes and guidelines for their unique needs and objectives. Many scholars, through books and journal articles, have also intended to unbox the complexities of ethical decision-making when using the internet both as a space and a tool for research in a wide range of disciplines. The vast quantity of content dedicated to ethical concepts, meanings, and dilemmas is a testament to the significance of this complex and ever-evolving field.

\section{Inclusion/exclusion criteria}

While there is a wealth of academic journals and books devoted to the subject of internet research, there is not as much coverage on gray literature that we argue to be equally as valuable when considering ethical research decisions. According to Auger (1998), this term broadly refers to non-commercially published materials, examples of which include reports, technical notes and specifications, conference proceedings, and supplementary publications, among others. The disciplinary guidelines included in our analysis fall under this category. Because of the fast-paced evolution of internet technologies and the adaptation required from the equally fluid field of the social sciences, we find that these sources are helpful in developing ethical decision-making in internet research. As Orton (2013) further contends, using these materials adds value to practice by providing free, accessible, and up-to-date content without the time and word count limits often involved in indexed and commercially published academic sources.

As the disciplinary guidelines we seek to analyse are not indexed in academic databases, a more general approach was employed. We used a combination of key terms "internet research," "ethics," "guidelines," and "associations" to 
conduct a wide search on the Google platform. This garnered approximately 10 million hits, which shows the interest in and relevance of the topic in current times. We narrowed down our search to the top 100 results, applying content, design, and quality criteria elaborated by Barry (1994), Savolainen and Kari (2006), and Xie et al. (2010).

Applying the content criterion, we only included documents that specifically discussed internet research ethics guidelines released by associations in non-commercial social sciences and those that mention internet, digital, or online research in the document title. We excluded entries that cover more general codes of professional research ethics that fall beyond the scope of this article as well as the otherwise insightful market research-focused ethical guidelines such as those released by the European Society for Opinion and Marketing Research (ESOMAR) in cooperation with the Global Research Business Network (GBRN) and the Council of American Survey Research Organisations (CASRO). Applying the design-related criterion, we only included document guidelines in report format, thus excluding relevant content in web page format. This process narrowed down our list to nine documents.

Applying the quality criterion, we further evaluated the documents based on the documents' currency and consensus. With regard to the former, we only included the latest version of internet research guidelines released by accredited associations from 2005 onwards. As Jones (2011) contends, the rapid evolution of the internet limit the understanding, and thus the content, of the documents released by associations to provide ethical guidance. For example, guidelines released before 2005 will not reflect the ethical issues in the use of social networking sites such as Facebook (ibid.). As a result of this process, only five out of the nine documents initially considered were included in the analysis, enumerated here from the latest date of publication: Ethics Guidelines for Internet-Mediated Research by the British Psychological Society (BPS) in 2017, Ethics Guidelines and Collated Resources for Digital Research by the British Sociological Association (BSA) in 2017, Ethical Guidelines for Internet Research by the National Committee for Research Ethics in the Social Sciences and the Humanities (NESH) in 2014, Ethical Decision-Making and Internet Research by the Association of Internet Researchers (AoIR) (Markham and Buchanan 2012), and Ethical Issues in Online Research by the British Educational Research Association (BERA) (Jones 2011). Applying the second quality criterion of consensus, we further confirmed the scientific impact of these five documents in the field of internet research ethics as they were frequently cited in the gray literature and academic sources we reviewed.

\section{Methods of analysis}

The documents were uploaded to the computer assisted qualitative data analysis software (CAQDAS) Atlas.ti for coding. As a first step, we agreed on a set of preliminary codes based on the three main principles of the Belmont Report and implemented a general keyword search on the document guidelines to check for the existence of sections that explicitly refer to these concepts. From this initial code set, all the co-authors then separately carried out an initial coding of a single document, the results of which were jointly discussed to detect the similarities and differences between our findings, the codes that emerged, and the strategy for their application in the next round of coding. We also drew on existing academic literature to substantiate the emergent codes that were not gleaned from the Belmont Report's invocations of each principle. After a consensus was reached, the first author implemented the second cycle of coding for the remaining guidelines. The most salient themes were then organised into categories and sub-categories and rearranged as the analysis progressed using theoretical coding (Saldaña 2009).

\section{Findings and discussion}

Following our analysis, we found that the guidelines analysed explicitly reflect the principles of respect for persons through informed consent and protection of vulnerable groups and beneficence through a balanced assessment of risks and harm as understood in the Belmont Report. On the other hand, the concept and applications of justice were rather implicit and showed a bigger shift from the original Report. These differences are summarized in Fig. 1 and will be discussed in more detail in the following sub-sections.

\section{General definitions and scope of internet research}

Out of the five guidelines analysed, only the BPS document reflects a definitive description of the activities and tools that can be categorised as internet research. BPS describes how internet-mediated research can involve data acquisition in the absence of face-to-face co-presence. This data can be quantitative (surveys and experiments) or qualitative (narratives) as well as reactive (participants interact with the materials such as online surveys and interviews) or nonreactive data (unobtrusively obtained data such as compilation of digital traces, hits, and analyses of found text). While lacking of an explicit definition of the activities involved in internet research, the AoIR, BERA, and NESH documents mention the role of internet both as a subject of research and a tool that spans from data collection to analysis and storage. 
Fig. 1 Extensions of the Belmont Report's principles based on the guidelines reviewed

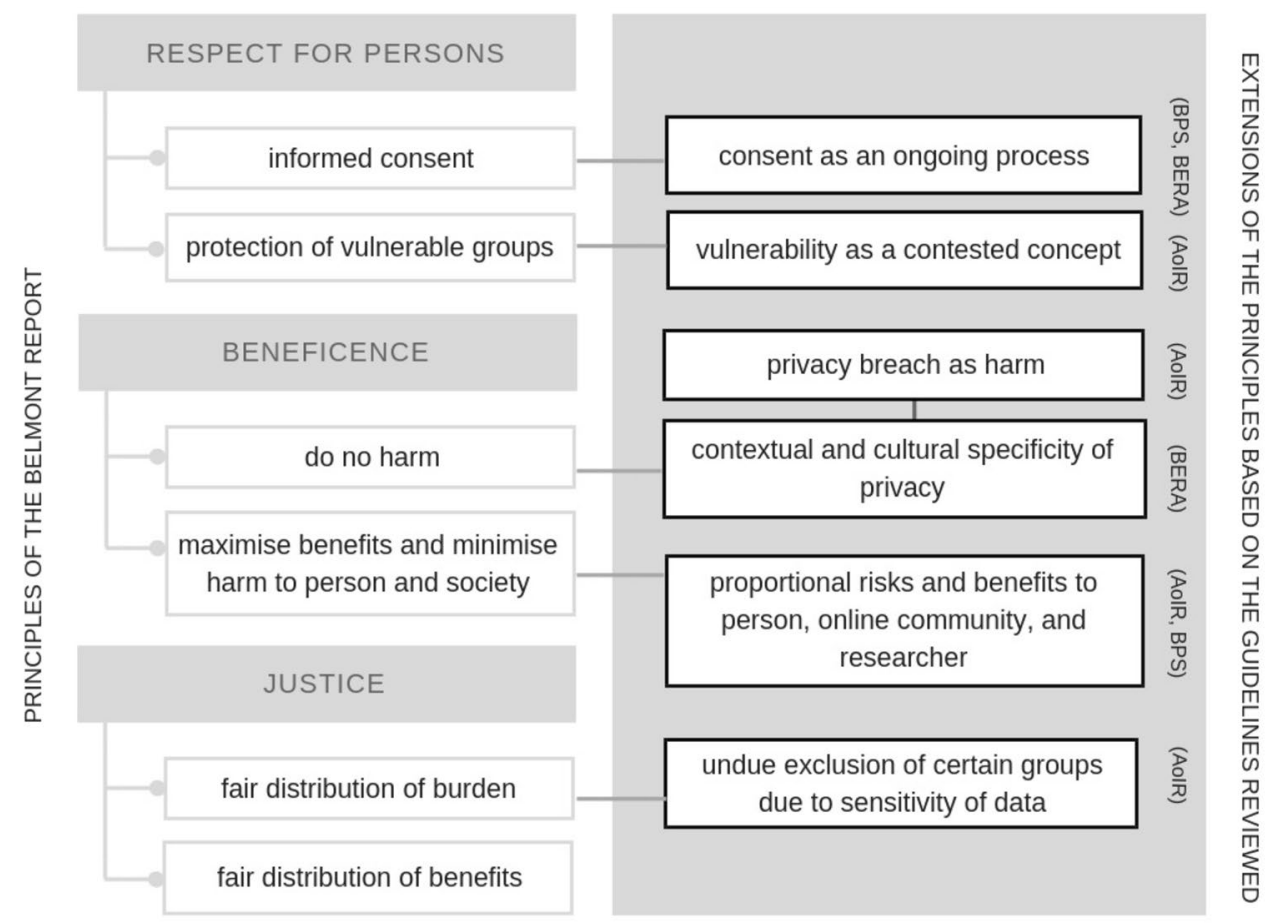

\section{Respect for persons}

The Belmont Report defines respect as the acknowledgment of participants' autonomous participation and the need to protect those with diminished autonomy through the conduct of informed consent. Following the analysis of the document guidelines on internet research, we have found that this is the most explicit principle reflected in the guidelines in terms of the applications and modifications in securing informed consent and protecting vulnerable groups, summarized in Fig. 2.

We will now turn to a more detailed discussion of the conceptualisations and practical applications of this first principle in the guidelines analysed.

\section{Informed consent}

The voluntariness of participation in ethically sensitive research is achieved by making the respondents fully aware of relevant information regarding the research, its objectives, the methods that will be adopted, and its potential risks and benefits. Seeking informed consent has been the most common way of ensuring that the ethical principle of voluntariness is practiced.

In traditional research concerning human subjects, consent is usually sought by having a respondent sign a document that outlines all relevant information including the risks and benefits implied. However, the current guidelines bring to light some new dilemmas and issues in seeking consent in online settings. Indeed, seeking written informed consent typical of traditional face-to-face research may require modifications when implemented online for a number of practical reasons. BSA provides a comprehensive summary of scenarios that are exempt from informed consent and confidentiality, quoting different sources. We suggest consulting the original document for a more detailed list. Meanwhile, Table 1 provides a synthesis of some scenarios wherein informed consent may be challenging to obtain or may actually be waived as identified in the five documents analysed: AoIR, BERA, BPS, BSA, and NESH.

Firstly, the volume of content and individual members in big online forums makes approaching each individual participant both impractical and time-consuming. As BERA suggests, the online and mediated nature of the process makes it more difficult for researchers to explain and for the participant to understand what he or she is consenting to. Informed consent may also be difficult, and sometimes even impossible, to gather from forum respondents whose contact details cannot be accessed (Jones 2011). Hoser and Nitschke (2010) regard this difficulty as a barrier for implementing this privacy measure, thus making the issue of informed consent more challenging in online settings.

Secondly, the wider variety and increased access to digital tools to researchers make unobtrusive data collection methods more accepted in academic research beyond its more common use in digital marketing. Unobtrusive methods such as data mining and undisclosed observation of online groups gather found and already existing data (Lee 2000) without disclosing the researcher's identity and without his or her involvement in communications (Eysenbach and Till 2001). 


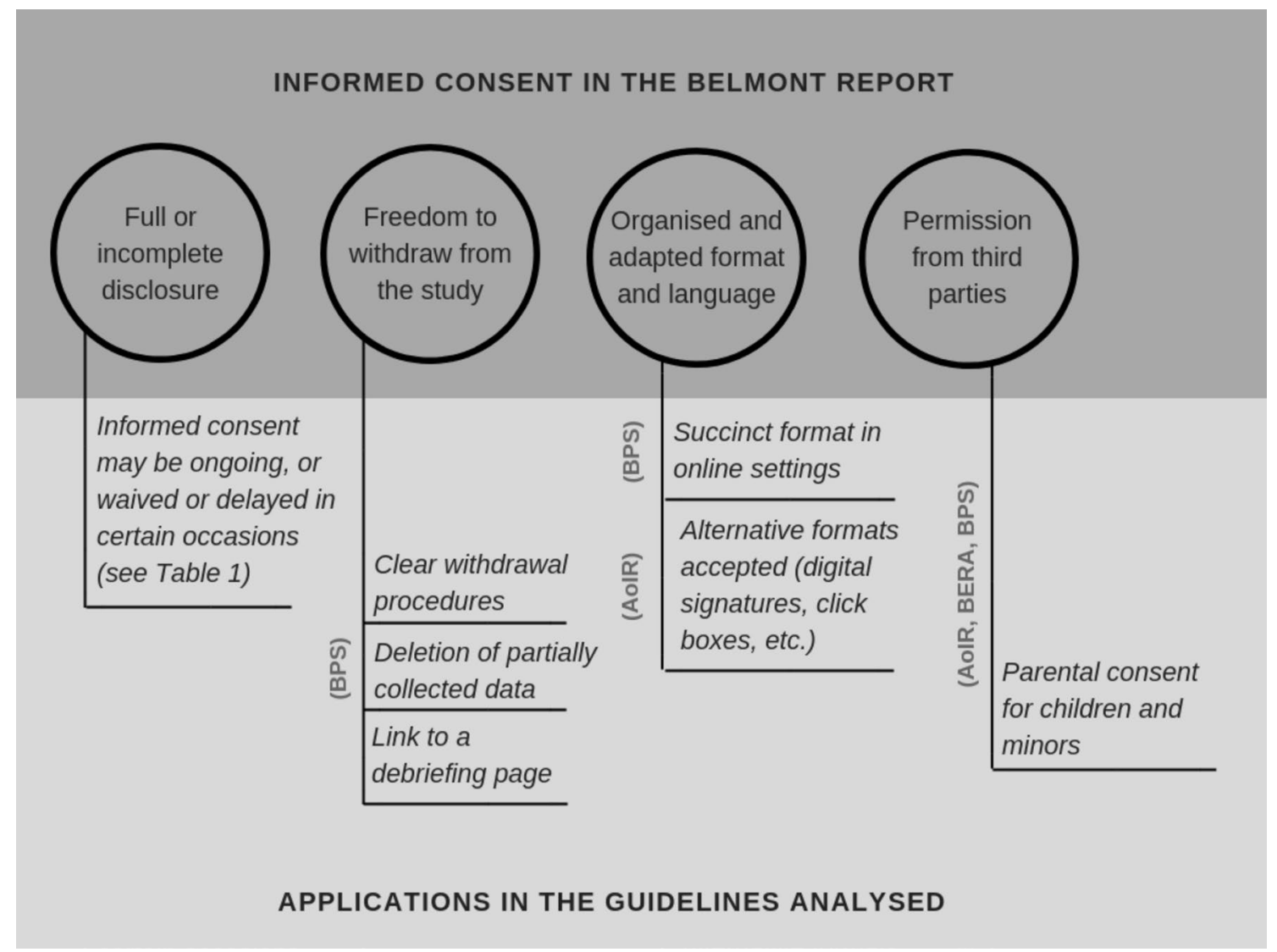

Fig. 2 Comparison of the application of the respect for persons principle as a function of informed consent between the Belmont Report and the guidelines analysed

Table 1 When and how to waive or delay informed consent based on disciplinary associations' guidelines

General approaches

Greater expectation of privacy (e.g. member-only access) raises the concern for informed consent (NESH 2014)

Ensure that study's scientific and social value outweighs its risks (BPS 2017)

Treat consent as an ongoing process (AoIR 2012; BSA 2017)

Specific scenarios and suggested approaches

Scenario 1: when participants' limited knowledge about the study improves its validity (BPS 2017) or reduces harm (NESH 2014)

Scenario 2: when informed consent cannot reasonably be sought or obtained during a research project (BERA/Jones 2011; AoIR 2012)

Scenario 3: when getting informed consent through traditional means becomes difficult or time-consuming (BERA/Jones 2011)

Scenario 4: when community membership configurations make the source of consent problematic (BPS 2017; NESH 2014)

Scenario 5: when people should be credited as authors (BSA 2017)
Request consent to use the data after the study's implementation (BERA/Jones 2011)

Ensure that withdrawal and debriefing measures are in place (BPS 2017)

Informed consent is sought at the time of reporting while allowing the research participant to decide how the results will be reported (BERA/Jones 2011; AoIR 2012)

Adapt the consent form by using digital signatures, consent tokens, and click boxes (AoIR 2012)

Indicate that survey completion grants the participant's consent (BPS 2017)

Approach community moderators to determine how and where to get the consent (BPS 2017)

Check for copyright, as some contexts and domains may be protected by copyright law (BPS 2017)

Treat opinions published by private individuals on the Internet as you would in traditional print media (Bassett and O'Riordan 2002 in BSA 2017)

Check Creative Commons guidelines (BSA 2017)

Inform authors if a link is made to the page (BPS 2017) 
In the same manner, the emerging field of learning analytics is subject to the same ethical dilemmas (Jones 2011).

The wider possibilities for data collection and the changing membership configurations in online communities make seeking informed consent less straightforward. Indeed, the propagation of both publicly accessible and membershiponly online forums raises the question of whether consent should be sought from the individual, the community, or both. In light of these changes, BPS suggests the role of community moderators and list owners when deciding on seeking the consent of a group, reaching out to them whenever possible to inquire about how to approach the informed consent process especially when it remains unclear whether the data involved is public or not. In relation to the textual nature of internet data, BPS, BSA, and AoIR also advise researchers to be aware of legal and copyright issues and to consider gaining permission from the page author or web hosting company when linking to publicly available personal websites and social network content as well as when using screenshots or images taken from the web. BSA points out the usefulness of being acquainted with the specifications of licenses such as Creative Commons when dealing with data online, although approaching the author or owner of the text works best to avoid harm. Strictly speaking, copyrighted content is not considered public domain.

While the BPS and NESH contend that informed consent should be strived for whenever possible, they also acknowledge that it may be waived in unique circumstances. BPS states that this can happen when there is reason to believe that there is no expectation of privacy among the group being studied or when the study's scientific and social value justifies undisclosed observation. NESH also asserts that researchers must be sensitive to the "integrity of context" especially that not all online users are aware that their private posts can be publicly accessible. One practical application of this is when accessing private groups. In this case, NESH declares that there is a higher expectation of privacy and, consequently, raises the concern for seeking valid consent and ensuring the anonymity of those involved.

Thirdly, informed consent in online settings may not always come in paper form. There are acceptable modifications suggested by AoIR, such as using digital signatures, virtual consent tokens, and click boxes. BPS also suggests that the completion of an online questionnaire can serve as an indication of consent, although the use of check boxes is always a good measure. It likewise recommends that consent through non-traditional means be simple enough to encourage the respondent to actually read its contents. A succinct presentation of the consent form in online settings is especially important to ensure that participants are thoroughly informed of the risks and benefits of the study and are not just randomly ticking boxes.
Lastly, researchers also have to be aware that opting to waive informed consent at the beginning of a study may actually be the most ethical approach in some cases, especially "when you want to present a specific case study or quote an individual or focus on a particular element" (Buchanan et al. 2010) or when withholding information (and thus informed consent) maintains the validity of the study (BPS). In such cases, BERA suggests that an ethical approach might be "to obtain informed consent when the project is at the point of reporting and the research subject can decide what is acceptable in relation to the way the research is to be reported" (n.p.), akin to what Roberts (2015) calls retrospective consent. According to BPS, the researcher should also put mechanisms in place to allow participants to be debriefed in the case of withdrawal from the study.

As suggested by the latest disciplinary associations' guidelines, ethically sensitive research conducted on the internet is an ongoing process. This is particularly true when seeking informed consent from participants wherein perceptions and expectations of privacy can change across groups and time. The disciplinary associations' guidelines reflected in this article argue for genuinely informed and valid consent that is not exclusively anchored in the traditional practice of signing a form. As Schneier (2000) and Orton-Johnson (2010) suggest, treating consent as a process rather than a product entails that we treat it not merely as a signed piece of paper. This can be achieved by the researcher making himself or herself available to the participant if any doubt regarding the research process arises (Whitehead 2007). As the tools for research expand, so should the ways in which consent can be sought and given.

\section{Voluntary withdrawal in online studies}

One of the aspects of the voluntariness of research participation in the Belmont Report is the freedom to withdraw from the study. BPS contends that compared to face-to-face studies, the risks are higher for internet-mediated research as withdrawal may happen without the researcher's knowledge. This consequently makes debriefing impossible and for some data to be collected and stored even after the withdrawal of participation.

As a measure of ethical practice, more specifically when carrying out online surveys, BPS suggests that participants should be made aware of the possibility for withdrawal of participation during and after the study and for deletion of data that has already been partially collected. Making withdrawal procedures clear is also good practice by providing a visible withdraw button that leads to a debrief page and the option to withdraw the partially collected data. In cases where the participant requests withdrawal from the study after it has been completed, also known as retrospective 
withdrawal, the researcher should be able to honor this taking into account the governing data protection laws. An identification code may be assigned to individual participants at the start of the study to facilitate possible withdrawal in the future, especially when dealing with large data sets.

In online focus groups, withdrawal from data collected through unobtrusive means may prove to be challenging. BPS describes how this type of data collection may include the withdrawing participant's identifiable information in the responses provided by other non-withdrawing participants and should thus be considered by researchers when storing and documenting data.

\section{The contested notion of vulnerability}

Protecting vulnerable groups is both an application of the principle of autonomy and beneficence, acting both as a gesture of respect for human dignity (Jones 2011) and a measure to minimize harm (BPS). AoIR, BERA, and BPS identify, albeit only in broad terms, which groups are deemed vulnerable in online settings. A point of convergence, however, is in the documents' declarations in the treatment of children and minors as vulnerable and in the need to seek parental or guardian consent. Both AoIR and BERA suggest that the principle of proportionality be applied, in that researching more vulnerable groups requires more care on the part of the researcher to protect them from harm. Conversely, AoIR contends that predicting which groups are vulnerable and the harm that may be induced as a result of the study is a challenging task. As a result, they propose a reflexive and negotiated approach to this principle by clarifying the various notions of vulnerability and harm in context.

\section{Beneficence}

The Belmont Report states that an ethical approach to research involves the principle of beneficience, wherein the researcher seeks to minimise harm and maximise benefits for human subjects by conducting a risk-benefit assessment. This principle remains salient in the guidelines analysed, with AoIR regarding ethical decision-making as a balancing act wherein decisions such as waiving informed consent and using deception are made through a careful and proportional consideration of their benefits and risks. The notion of risk reflected in the Belmont Report basically alluded to psychological or physical pain or injury, although it likewise acknowledges legal, social, and economic harm that might result from one's participation in research.

While several scholars contend that the online settings usually involve minimal risk (Kraut et al. 2004), others argue that and both online and offline settings require just equals amounts of sensitivity (Eynon et al. 2008). However, the guidelines analysed revealed some nuances in the ethical conduct of internet research in relation to the observance of the principle of beneficence, summarized in Fig. 3. As Buchanan (2011) suggests, the very existence of internetspecific guidelines conveys that there are new challenges arising from the emergence of this new research space and medium.

In the subsequent sections, we will discuss specific beneficence-related considerations outlined in the document guidelines analysed.

\section{Privacy breach as harm: contextuality and culture-specificity of expectations of privacy}

Following the analysis of the five disciplinary associations' guidelines, it was found that protection of privacy is one of the most salient themes in this principle, thus reinforcing the assertion regarding the distinct nature of online research. Although privacy is not explicitly reflected in the original Belmont Report, it has since become a pressing concern and a source of potential harm that researchers should seek to mitigate. BPS suggests that the risks are greater for privacy breaches that are beyond the researcher's control. This is corroborated by Kraut et al. (2004), describing how online studies generally involve minimal harm but can lead to negative results when data is misused. As Boyd (2010) points out, the greater risk to privacy in online settings is due to the fact that internet-mediated data and tools are persistent, searchable, scalable, and replicable.

As AoIR suggests, harm may manifest not only in physical but also in social, psychological, and economic terms. One of the prevalent ways that psychological and economic harm is induced in online social science studies is when one's reputation is damaged due to a privacy breach (Gaiser 2008; Rasmussen 2008) and leads to the loss of a job. As such, all the guidelines analysed highlight the importance of researchers' awareness of the specificities of the context in which the research is being undertaken and the participants' corresponding expectations of privacy, especially given the global reach and cultural diversity found in internet settings (BPS). AoIR and BSA refer to this as a process-based and dialogic approach to ethics where the public or private nature of data and interaction spaces is subject to ongoing negotiation.

To address and mitigate privacy-related forms of harm, the analysed documents suggest three approaches. First, researchers are advised to keep the participants' data and responses anonymous as well as to ensure that their identities cannot be tracked by using pseudonyms (NESH) and vignettes (BERA), releasing only general or conglomerated data (BERA), and using paraphrased statements (BPS). Secondly, BPS advises researchers to be conscientious of how they store and transfer data, with email correspondence and non-encrypted data storage being more prone to privacy 


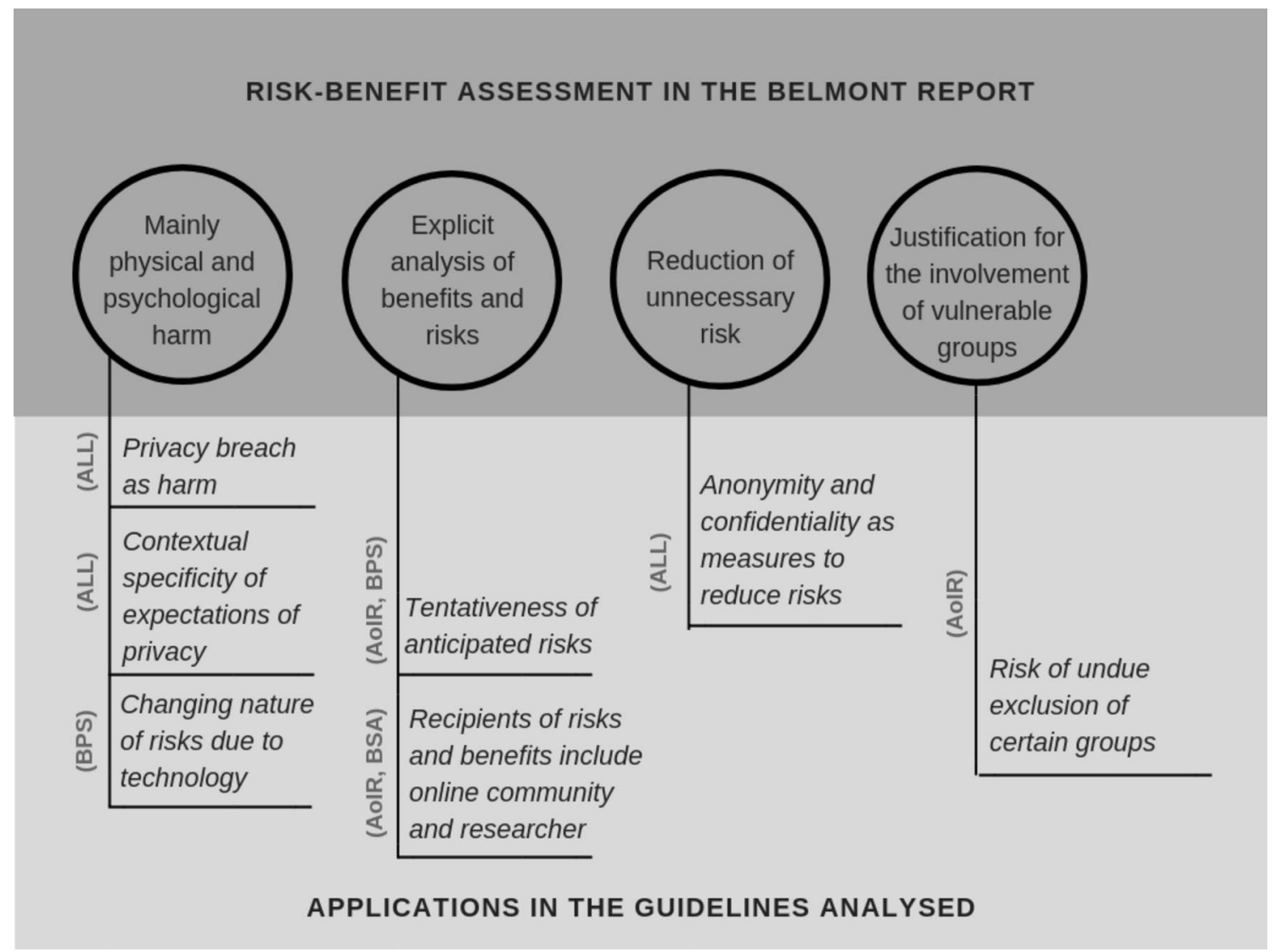

Fig. 3 Comparison of the application of the beneficence principle as a function of risk-benefit assessment between the Belmont Report and the guidelines analysed

breach. Lastly, while researchers should strive to exhaust all measures to protect the participants' identities, they must also be wary of guaranteeing complete confidentiality when in fact it cannot be assured (BPS) due to the text-based and archiving capacities of internet technologies.

However, the AoIR presents a natural caveat to this: the risk cannot be always assumed in the varied contexts and changing meanings that the internet allows. This is exemplified in Bassett and O'Riordan's (2002) study on an LGBT website, wherein an otherwise marginalised population sought for visibility by publicising sensitive data. Discarding the data altogether because of its sensitive nature can lead to further marginalisation. Indeed, "a rhetoric of "protection' may result in furthering the unequal power relations of media production by blocking full representation of alternative media" (Bassett and O'Riordan 2002, p. 244). Researchers thus need to be aware that some sources providing sensitive data should not be elided just because the original contributors contact details, and therefore consent, are not readily available.

\section{Risks and benefits for whom? Emerging issues in internet research}

Another notable departure from the interpretation of beneficence in the original Belmont Report is the changing conceptions regarding the subjects and recipients of risk when conducting research. In addition to the concern for participants' welfare and the benefits gained by the wider society, the guidelines analysed extend their interpretations of beneficence to online communities and the researchers themselves. The shift of language from society to communities reflect the underlying premise of trust that characterizes many virtual social spaces. NESH also points out that social and cultural movements that function on norms of openness and freedom abound in the online setting, thus making it imperative to consider adopting a sharing mindset when it comes to communicating the outcomes and benefits of research to the participants. The safety and interest of the researcher are likewise considered in the current interpretation of ethical internet research. According to the BSA, researchers should be wary about assuming a vulnerable position and being at the receiving end of abuse given their online presence. 


\section{Justice}

In relation to the protection of vulnerable groups in the first principle of respect for persons, the Belmont Report interprets justice to be a fair distribution of the risks and benefits of research and exemplifies its application in the selection of subjects. As we had previously mentioned, our analysis revealed that the applications of the justice principle are the least explicit of all three principles in the guidelines. The absence of the nomenclature used in the Belmont Report may be due to the fact that the documents seek to provide guidance at a practical level. Secondly, research often involves overlapping applications of these principles wherein elements of the justice principle are subsumed in others, such as in the case of protecting vulnerable groups as a function of respect for persons. Thirdly, the implicitness of this principle may also be influenced by how different the manifestation of the principle of justice is in the textual (Bassett and O'Riordan 2002), and oftentimes social and participatory, nature of online research from the Belmont Report's biomedical roots. In online research settings, the focus is less likely on treatments and interventions and is instead geared towards information retrieval and group participation. Lastly, it may well be that the very ethical configurations that frame research in general are changing, making the issue of fairness reflected in the Belmont Report insufficient in defining an ethical research practice. Because of these reasons, we drew on existing literature to illuminate the implicit interpretations of justice and identified some gaps in its formulation, summarized in Fig. 4 below and which we will discuss in more detail in the following sub-sections.

\section{Protectionist versus inclusive ethics in internet research}

We found an overarching protectionist stance in the Belmont Report when approaching vulnerable groups that runs parallel with Shore's (2006) contention. This is understandably so, considering the fact that the conceptualisation of justice in the original Belmont Report was largely influenced by the exploitation of certain groups in the nineteenth and twentieth centuries for biomedical research. The prevailing social conditions at that time prompted an approach to ethics that was primarily concerned with avoiding the danger of recruiting

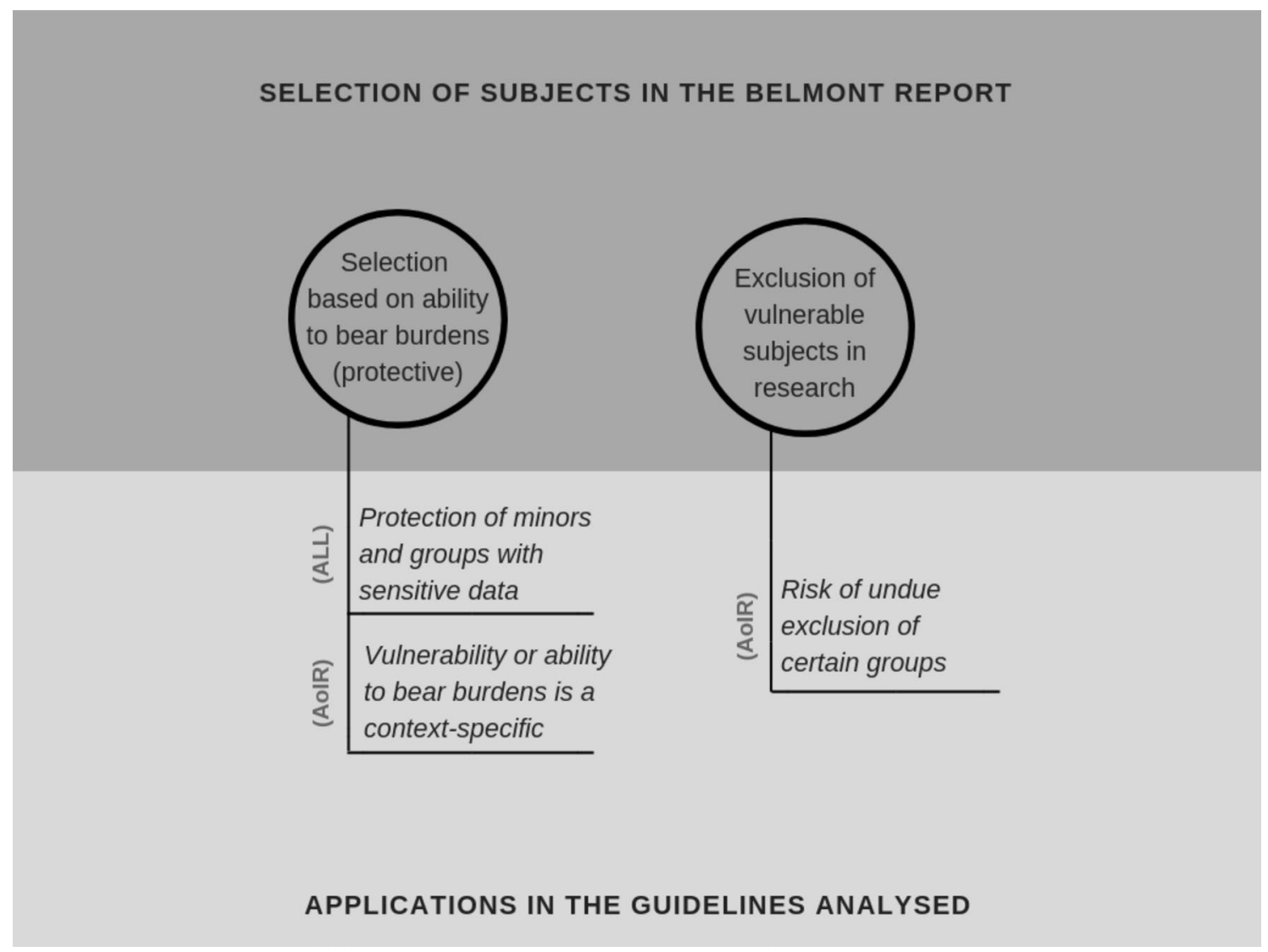

Fig. 4 Comparison of the application of the justice principle as a function of subject selection between the Belmont Report and the guidelines analysed 
certain profiles of individuals (e.g. ward patients, prisoners, rural black men, etc.) for experiments and clinical trials without them receiving the benefits of the research findings (National Commission 1979; Shore 2006). As a result, the Belmont Report pushed for a hierarchical selection procedure when recruiting subjects, opting for exclusion of disadvantaged groups to avoid further burdening them.

However, our analysis of the current internet research guidelines revealed a combination of protective and inclusive approach to the principle of justice. This is corroborated by Shore (2006), pointing out that the subsequent extensions of the Belmont Report from its original conception involve an awareness of the risk of certain groups' exclusion. Together with making sure that groups with highly sensitive data are protected, especially as they relate to the interest of children and minors, AoIR asks whether these groups are being excluded from research due to the difficulties in securing ethical approval. Even BPS acknowledges that some political activist groups may be open to nonanonymous publications of their information and responses in line with their mission as a community. In this regard, we see how the discourse is shifting from an exclusionary to an inclusionary language in the application of justice. This is consistent with Bassett and O'Riordan's (2002) study mentioned in the previous section, which provides a clear example of how an outright avoidance of what researchers would perceive as sensitive data can cause some voices to be silenced. They argue that academic researchers need to take into account the internet's various uses for a wide range of groups as well as the cultural production and visibility that it could provide for traditionally marginalised populations. Walther (2002) and Kozinets (2015) also point out that both the textual and spatial qualities of internet use allow for different methodological approaches, such as a focus on the linguistic features of the text in the former and the more observational or anthropologic studies in the latter. As such, what counts as ethical decision-making would differ in these scenarios. Ultimately, researchers should be careful about swaying too far on the protectionist side when carrying out online research.

\section{Shifting ethical frameworks}

The inclusion-exclusion tension that surfaced in our analysis reveals a shift in the general understanding of what is deemed ethical in research. While the Belmont Report subscribes to fairness of distribution as an application of justice, the guidelines analysed are geared towards culturally sensitive and dialogic approaches that are open to negotiation. All the guidelines have retained the need to protect the welfare of those involved in the research, albeit an overarching theme is an appreciation of the multiplicity of meanings and expectations of individuals and communities online as well as the diversity of geographies, cultures and contexts in which they operate. Both the AoIR and the BSA promote a case-based or situational approach to ethics against a backdrop of changing technologies and possible uses of data found online. The reflexivity that characterizes postmodern thinking has likely contributed to the changing definitions of harm and vulnerability that affects researchers' ethical decision-making. Thus, we cannot readily assume that groups that are traditionally considered vulnerable would want to be excluded from research.

\section{Justice: gaps and future directions}

We have found this principle to be the least developed in the documents analysed that, while not necessarily leading to unethical research, is something that warrants attention and explicitness in the future versions of these guidelines. The distributive justice that characterizes the Belmont Report (Longres and Scanlon 2001) seems insufficient to cover the relational justice requirements of community research that abound on the internet (Shore 2006). In practice, researchers are tasked to strike a balance between protection and effective participation. Adopting this approach opens up the possibility for researchers to what Hine (2005) identifies as political action through research, whose function is "to sidestep, transform, highlight, or reinvent some traditional political transformations, identities and inequalities" (p. 242). A widely reaching platform such as the internet can provide this leverage if used wisely.

Having considered the strong potential of the internet for democratization (Mann and Stewart 2011), it would be helpful if the current guidelines could alert researchers to the various forms of sampling and recruitment issues inherent in online research, an element that appears to be lacking in the guidelines' current form. Several authors have alluded to the internet's non-representativeness of the general population (Eysenbach and Wyatt 2002; Im and Chee 2011) Therefore, the challenge that researchers are confronted with involves deciding if the internet is an appropriate methodological tool and, depending on the research design, whether or not extrapolating research findings to the general population is appropriate (Walther 2002).

\section{Summary and conclusions}

In this paper, we attempted to illuminate the conceptualisations and applications of the Belmont Report's ethical principles of respect for persons, beneficence, and justice in internet-mediated research through a document analysis of five recent disciplinary guidelines with the greatest scientific impact in the field of social sciences. In general, the disciplinary guidelines' discourse is marked by a shift from 
a prescriptive to a more situational and dialogic approach to internet-mediated research ethics. This may alleviate the pains of a social science researcher who finally encounters forgiving ways to approach a research practice characterized by fast-paced technological advances and fuzzy social boundaries. It was also found that the existing guidelines reflect the core principles that originally appeared in the Belmont Report, albeit in varying degrees and with a nuanced understanding of its applications. The adapted informed consent procedures, increased awareness of risks to privacy, and the rather implicit reference to justice-related issues are some of the highlighted differences with the Belmont Report's contents.

There are several recommendations we wish to make in the ongoing development of internet research ethics in light of this analysis. Firstly, the disciplinary associations' guidelines, with the exception of BPS's, were lacking in the definitive description or categorisation of the activities and tools involved in internet research. We argue that reflecting a clearer typology of what constitutes internet research in the document guidelines themselves would lead to a better understanding of their uses and intended applications for social science researchers. As our understanding of the typologies and activities involved in internet research widens, it will be a worthwhile endeavor for disciplinary associations to continue developing ways to draw the distinction between approaches that focus on the spatiality or textuality of internet-mediated research as they entail quite different ethical implications. Secondly, we find opportunities for enhancement in terms of making justice-related conceptualisations and applications explicit in the guidelines. This may be done by bringing researchers' attention to the possibility of unintentionally excluding certain groups and the ensuing loss of benefits for them as well as the sampling issues on the internet on the basis of user demographics. Consequently, researchers should be particularly attuned to the social impact of their studies as well as the internet's emancipatory potential for underserved groups-something that is otherwise lost when adopting unnecessarily stringent ethical standards.

While we acknowledge that this analysis has put forward a rather field-specific coverage of ethical issues, we believe that critically examining the current version of ethical guidelines released by relevant disciplinary associations proves valuable in the often complicated process of ethical decision-making in research. By outlining the gaps and providing recommendations, our hope is to contribute to promoting respect, beneficence, and justice in their varied forms - tensions notwithstanding — when utilising an everevolving medium that is the internet.

Acknowledgements This work was supported by the University of Deusto Research Training Grant (FPI 2016).

\section{Compliance with ethical standards}

Conflict of interest The authors declare that they have no conflict of interest.

Research involving human participants and/or animals This article does not contain any studies with human participants or animals performed by any of the authors.

Open Access This article is distributed under the terms of the Creative Commons Attribution 4.0 International License (http://creativeco mmons.org/licenses/by/4.0/), which permits unrestricted use, distribution, and reproduction in any medium, provided you give appropriate credit to the original author(s) and the source, provide a link to the Creative Commons license, and indicate if changes were made.

\section{References}

Armstrong, R. (2003). Human subjects research and the Internet: Ethical dilemmas. Research Review. University of Texas Office of Research Administration. Retrieved from https://research.utexa s.edu/wp-content/uploads/sites/3/2015/10/ehtical_dilemmas_inter net.pdf.

Auger, C. (1998). Information sources in grey literature (4th ed.). London: Bowker-Saur.

Barry, C. (1994). User-defined relevance criteria: An exploratory study. Journal of the American Society for Information Science, 45(3), 149-159.

Bassett, E. H., \& O'Riordan, K. (2002). Ethics of Internet research: Contesting the human subjects research model. Ethics and Information Technology, 4, 233-247.

Beauchamp, T., \& Childress, J. (1994). Principles of biomedical ethics. New York: Oxford University Press.

Boyd, D. (2010). Social network sites as networked publics: Affordances, dynamics, and implications. In Z. Papacharissi (Ed.), Networked self: Identity, community, and culture (pp. 39-58). New York: Routledge

British Psychological Society. (2017). Ethics guidelines for Internetmediated research. INF206/04.2017. Leicester: British Psychological Society.

British Sociological Association. (2017). Ethics guidelines and collated resources for digital research: Statement of ethical practice annexe. Retrieved from https://www.britsoc.co.uk/media/24309/ bsa_statement_of_ethical_practice_annexe.pdf.

Buchanan, E. (2011). Internet research ethics: Past, present, and future. In M. Consalvo \& C. Ess (Eds.), The handbook of internet studies. Oxford: Wiley. https://doi.org/10.1002/9781444314861.ch5.

Buchanan, E., Markham, A., \& Ess, C. (2010). Ethics and internet research commons: Building a sustainable future. Association of Internet Researchers 11th Annual Conference Workshop. Gottenburg, Sweden.

Buchanan, E., \& Zimmer, M. (2018). Internet research ethics. In E. N. Zalta (Ed.), The Stanford encyclopedia of philosophy. Stanford: Stanford University;(Spring 2018 Edition).

Castells, M. (2001). The Internet galaxy: Reflections on the Internet, business, and society. Oxford: Oxford University Press.

Convery, I., \& Cox, D. (2012). A review of research ethics in internetbased research. Practitioner Research in Higher Education, 6(1), 50-57.

Dench, S., Iphofen, R., \& Huws, U. (2004). An EU code of ethics for socio-economic research. Brighton: The Institute for Employment Studies. 
ESOMAR \& GBRN. (2015). Global guideline: Online research. Retrieved from https://www.esomar.org/uploads/public/knowl edge-and-standards/codes-and-guidelines/ESOMAR-GRBNOnline-Research-Guideline-October-2015.pdf.

Eynon, R., Fry, J., \& Schroeder, R. (2008). The ethics of internet research. In R. Lee, N. Fielding \& G. Blank (Eds.), The Sage handbook of online research methods. London: Sage.

Eysenbach, G., \& Till, J. (2001). Ethical issues in qualitative research on internet communities. British Medical Journal, 323, 1103-1105.

Eysenbach, G., \& Wyatt, J. (2002). Using the Internet for surveys and health research. Journal of Medical Internet Research, 4(2), e13.

Frankel, M., \& Siang, S. (1999). Ethical and legal issues of human subjects research on the Internet-Report of a workshop June 10-11. Retrieved from http://shr.aaas.org/projects/human_subje cts/cyberspace/ report.pdf.

Gaiser, T. J. (2008). Online focus groups. In R. Lee, N. Fielding \& G. Blank (Eds.), The Sage handbook of online research methods. London: Sage.

Gunsalus, C., Bruner, E., Burbules, N., Dash, L., Finkin, M., Goldberg, J., Greenough, W., Miller, G., \& Pratt, M. (2006). Mission creep in the IRB world. Science, 312(5779), 1441. https://doi.org/10.1126/ science. 1121479.

Hewson, C., Yule, P., Laurent, D., \& Vogel, C. (2003). Internet research methods: A practical guide for the social and behavioural sciences. London: Sage.

Hine, C. (2005). Internet research and the sociology of cyber-socialscientific knowledge. The Information Society, 21(4), 239-248. https://doi.org/10.1080/01972240591007553.

Hine, C. (2011). Internet research and unobtrusive methods. Social Research Update, 61, 1.

Hoser, B., \& Nitschke, T. (2010). Questions on ethics for research in the virtually connected world. Social Networks, 32(3), 180-186. https://doi.org/10.1016/j.socnet.2009.11.003.

Im, E., \& Chee, W. (2011). Recruitment of research participants through the internet. Computer, Informatics, Nursing, 22(5), 289-297.

Jones, C. (2011). Ethical issues in online research. British Educational Research Association online resource. Retrieved from https:// www.bera.ac.uk/researchers-resources/publications/ethical-issue s-in-online-research.

Kozinets, R. (2015). Netnography redefined. London: SAGE.

Kraut, R., Olson, J., Banaji, M., Bruckman, A., Cohen, J., \& Cooper, M. (2004). Psychological research online: Report of board of scientific affairs' advisory group on the conduct of research on the internet. American Psychologist, 59(4), 1-13.

Lee, R. M. (2000). Unobtrusive methods in social research. Buckingham: Open University Press.

Longres, J., \& Scanlon, E. (2001). Social justice and the research curriculum. Journal of Social Work Education, 37(3), 447-463.

Mann, C., \& Stewart, F. (2011). Internet communication and qualitative research: A handbook for research online. London: SAGE.

Markham, A., \& Buchanan, E. (2012). Ethical decision-making and internet research. Recommendations from the AoIR Ethics Working Committee (Version 2.0). Retrieved from http://www.aoir.org.

Markham, A., \& Buchanan, E. (2015). Internet research: Ethical concerns. International Encyclopedia of the Social \& Behavioral Sciences, 12, 606-613.

Moreno, M. A., Goniu, N., Moreno, P. S., \& Diekema, D. (2013). Ethics of social media research: Common concerns and practical considerations. Cyberpsychology, Behavior and Social Networking, 16(9), 708-713. https://doi.org/10.1089/cyber.2012.0334.
National Commission for the Protection of Human Subjects of Biomedical and Behavioral Research. (1979). The Belmont Report: Ethical principles and guidelines for the protection of human subjects of research. Bethesda, MD: National Commission for the Protection of Human Subjects of Biomedical and Behavioral Research.

Office for Human Research Protections. (2016). The Belmont Report. Retrieved from https://www.hhs.gov/ohrp/regulations-and-polic y/belmont-report/index.html.

Orton, C. (2013). Using the grey literature to enhance research and practice in continuing education for health professionals. Journal of Continuing Education in the Health Professions, 33(1), 1-3.

Orton-Johnson, K. (2010). Ethics in online research: Evaluating the ESRC Framework for research ethics categorization of risk. Sociological Research Online, 15(4), 13.

Parker, C., Saundage, D., \& Lee, C. (2011). Can qualitative content analysis be adapted for use by social informaticians to study social media discourse? A position paper. ACIS 2011 Proceedings. Paper 90. Retrieved from http://aisel.aisnet.org/acis2011/90.

Platt, J. (2015). Social science learned societies and professional associations. International Encyclopedia of the Social \& Behavioral Sciences, 22, 674-678.

Rasmussen, K. B. (2008). General approaches to data quality and internet-generated data. In R. Lee, N. Fielding \& G. Blank (Eds.), The Sage handbook of online research methods. London: Sage.

Roberts, L. (2015). Ethical issues in conducting qualitative research in online communities. Qualitative Research in Psychology, 12(3), 314-325. https://doi.org/10.1080/14780887.2015.1008909.

Saldaña, J. (2009). The coding manual for qualitative researchers. Los Angeles: SAGE.

Savage, M., \& Burrows, R. (2007). The coming crisis of empirical sociology. Sociology, 41(5), 885-899.

Savolainen, R., \& Kari, J. (2006). User-defined relevance criteria in web searching. Journal of Documentation, 62(6), 685-707.

Schneier, B. (2000). Secrets and lies: Digital security in a networked world. New York: Wiley.

Shore, N. (2006). Re-conceptualizing the Belmont Report: A community-based participatory research perspective. Journal of Community Practice, 14(4), 5-26. https://doi.org/10.1300/J125v14n04 -02.

Siebert, J., Plattner, S., \& Rubin, P. (2002). How (not) to regulate social and behavioral research. Professional Ethics Report, 15(2), 1-3.

Townsend, L., \& Wallace, C. (2016). Social media research: A guide to ethics. Economic and Social Research Council. Retrieved from http://www.gla.ac.uk/media/media_487729_en.pdf.

Vilar Martín, J. (2013). Cuestiones éticas en la educación social: Del compromiso político a la responsibilidad en la práctica profesional. Barcelona: UOC.

Walther, J. B. (2002). Research ethics in Internet-enabled research: Human subjects issues and methodological myopia. Ethics and Information Technology, 4(3), 205-206.

Whitehead, L. C. (2007). Methodological and ethical issues in Internetmediated research in the field of health: An integrated review of the literature. Social Science and Medicine, 65, 782-791.

Wilson, R. E., Gosling, S. D., \& Graham, L. T. (2012). A review of Facebook research in the social sciences. Perspectives on Psychological Science, 7(3), 203-220. https://doi.org/10.1177/17456 91612442904.

Xie, I., Benoit III, E., \& Zhang, H. (2010). How do users evaluate individual documents? An analysis of dimensions of evaluation activities. Information Research, 15(4), papercolis723. 